\title{
BMJ Open Association between psychosocial factors at work and health outcomes after retirement: a protocol for a systematic review and meta-analysis
}

Kotaro Imamura, ${ }^{1}$ Akizumi Tsutsumi, ${ }^{2}$ Yumi Asai, ${ }^{1}$ Hideaki Arima, ${ }^{1}$ Emiko Ando, ${ }^{3}$ Akiomi Inoue, ${ }^{2}$ Reiko Inoue, ${ }^{2}$ Mai Iwanaga, ${ }^{4}$ Hisashi Eguchi, ${ }^{2}$ Yasumasa Otsuka, ${ }^{5}$ Yuka Kobayashi, ${ }^{1}$ Asuka Sakuraya, ${ }^{6}$ Natsu Sasaki, ${ }^{1}$ Kanami Tsuno, ${ }^{7}$ Ayako Hino, ${ }^{8}$ Kazuhiro Watanabe, ${ }^{1}$ Akihito Shimazu, ${ }^{9}$ Norito Kawakami ${ }^{\circ 1}$

To cite: Imamura K, Tsutsumi A, Asai Y, et al. Association between psychosocial factors at work and health outcomes after retirement: a protocol for a systematic review and meta-analysis. BMJ Open 2019;9:e030773. doi:10.1136/ bmjopen-2019-030773

- Prepublication history and additional material for this paper are available online. To view these files, please visit the journal online (http://dx.doi. org/10.1136/bmjopen-2019030773).

Received 01 April 2019 Revised 13 August 2019 Accepted 13 August 2019

Check for updates

(C) Author(s) (or their employer(s)) 2019. Re-use permitted under CC BY-NC. No commercial re-use. See rights and permissions. Published by BMJ.

For numbered affiliations see end of article.

Correspondence to Professor Norito Kawakami; nkawakami@m.u-tokyo.ac.jp

\section{ABSTRACT}

Introduction The world's population is rapidly ageing, and health among older people is thus an important issue. Several previous studies have reported an association between adverse psychosocial factors at work before retirement and postretirement health. The objective of this systematic review and meta-analysis is to examine the association between psychosocial factors at work and health outcomes after retirement, based on a synthesis of well-designed prospective studies.

Methods and analysis The participants, exposures, comparisons and outcomes of the studies in this systematic review and meta-analysis are defined as follows: (P) people who have retired from their job, (E) presence of adverse psychosocial factors at work before retirement, (C) absence of adverse psychosocial factors at work before retirement and $(0)$ any physical and mental health outcomes after retirement. Published studies were searched using the following electronic databases: MEDLINE, EMBASE, PsycINFO, PsycARTICLES and Japan Medical Abstracts Society. The included studies will be statistically synthesised in a meta-analysis to estimate pooled coefficients and $95 \% \mathrm{Cls}$. The quality of each included study will be assessed using the Risk Of Bias In Non-randomised Studies-of Interventions. For the assessment of meta-bias, publication bias will be assessed by using Egger's test, as well as visually on a funnel plot. Heterogeneity will be assessed using the $\chi^{2}$ test with Cochran's $Q$ statistic and $I^{2}$.

Ethics and dissemination Results and findings will be submitted and published in a scientific peer-reviewed journal and will be disseminated broadly to researchers and policy-makers interested in the translatability of scientific evidence into good practices.

PROSPERO registration number CRD42018099043.

\section{INTRODUCTION}

The population of the world is rapidly ageing. The United Nations reported that the global population of those aged 60 or above is growing at a rate of $3.26 \%$ per year, and the number of persons in this age group is
Strengths and limitations of this study

- This will be the first systematic review and meta-analysis to show integrated evidence for associations between psychosocial factors at work before retirement and postretirement health conditions.

- The findings would contribute to prevention of chronic conditions and promotion of health and well-being of older adults after retirement and to achieve active ageing.

- Practically, the results of this study could facilitate implementation of appropriate intervention for workers who have been exposed to specific adverse psychosocial factors at work.

- One major limitation is that this study will include mostly observational studies and the findings may be biassed by potential confounds.

projected to be 2.1 billion $(21.5 \%)$ by $2050 .{ }^{1}$ Within this context, health and well-being among older people is focused on important issues. $^{2-6}$ To respond to this global challenge, WHO has developed a policy framework of 'Active Ageing', which optimises opportunities for health, participation and security in order to enhance the quality of life of older people. ${ }^{7}$ The life course approach adopted in WHO Active Ageing policy framework ${ }^{7}$ is an approach intended to maintain and prevent the deterioration of functional capacity of older people. ${ }^{8}$ Determinants of health in older age are established in early childhood, even before birth, and influenced by conditions experienced throughout life. Therefore, it is important to apply the life course perspective to considering the dynamic process and multidimensional nature of health and wellbeing in adults and elderly. ${ }^{9}$ Some reviews have reported that the risk factors including some sociodemographic factors, poor mental 
health, chronic physical disease burden and adverse lifestyle habits and behaviours for functional decline and mortality increased in the elderly. ${ }^{10-12}$ These risk factors are important targets for health promotion. Especially, it is strategically important to reduce potentially modifiable risk factors in early life and across the life course. ${ }^{11}$

Work, including employment and working conditions, has been recognised as an important social determinant of health in the working age population. ${ }^{13}$ However, work also may be an important life course determinant of health as a person ages. Recently, the association of working conditions and employment has received attention as a social determinant of health status for older people (ie, after retirement). For instance, while the overall impact of retirement (including early or voluntary retirement) and health has been reported small and inconsistent, ${ }^{15}$ people who worked in white-collar jobs have tended to have a more beneficial health effect after retirement than those who worked in blue-collar jobs. ${ }^{16}$ There is a possibility that employment status and working conditions at the working age could affect health at an older age after retirement.

Psychosocial factors at work are well-known determinants of health on working population. The Joint International Labour Organization/WHO Committee on Occupational Health has defined psychosocial factors at work as 'interactions between and among work environment, job content, organisational conditions and workers' capacities, needs, culture, personal extrajob considerations that may, through perceptions and experience, influence health, work performance and job satisfaction'. ${ }^{17}$ Several previous studies reported an association between adverse psychosocial factors at work and postretirement health. Some longitudinal studies reported that work-related stress (ie, high job strain or high job demands and lack of control) as defined in the job demand-control model ${ }^{18}$ was associated with self-reported health problems in old age. ${ }^{1920}$ For mental health, previous longitudinal studies reported a significant association between several adverse psychosocial factors at work (ie, high job strain, high demand, low control, low reward and low support) and depressive symptoms after retirement. ${ }^{2122}$ In addition, a longitudinal study reported that lack of job control was associated with poorer levels of episodic memory at and following retirement. ${ }^{23}$ To reduce potentially modifiable risk factors across the life course, an effective strategy might be to target on improving psychosocial factors at work before retirement. However, there is no systematic review or meta-analysis that has gathered evidence from well-designed prospective cohort studies on the impact of adverse psychosocial factors at work on health outcomes after the retirement.

\section{Objectives}

The objective of this systematic review and meta-analysis is to examine the association between psychosocial factors at work and health outcomes after retirement, based on a synthesis of well-designed prospective studies. The results of this study could expand the current evidence regarding the effect of psychosocial factors at work on worker health ${ }^{2425}$ to include their impact on health in older people after retirement. In addition, the results of this study could contribute to a better understanding of the quality of employment on health in later life, and the development of a new perspective on the life-course strategy for promoting active ageing. ${ }^{7}$

\section{METHODS AND ANALYSIS}

\section{Study design}

This is a systematic review and meta-analysis protocol of prospective studies, according to the Preferred Reporting Items for Systematic Reviews and Meta-Analyses protocols guideline. ${ }^{26}$ The systematic review and meta-analysis will be reported according to the Meta-analysis Of Observational Studies in Epidemiology guideline. ${ }^{27}$

\section{Participants, exposures, comparisons and outcomes and eligibility criteria of this study}

The participants, exposures, comparisons and outcomes (PECO) of the studies in this systematic review and meta-analysis are defined as follows:

(P) People who have retired from their job.

(E) Presence of adverse psychosocial factors at work before retirement.

(C) Absence of adverse psychosocial factors at work before retirement.

(O) Any physical and mental health outcomes after retirement.

The adverse psychosocial factors at work include a wide range of task and organisational characteristics, working conditions and workplace interactions, such as job strain, effort-reward imbalance, working hours, shift work, low social support and other organisational-level factors.

Inclusion criteria are as follows:

1. Studies that included participants who were working as of the baseline survey period.

2. Studies that assessed adverse psychosocial factors at work before retirement as exposure variables at baseline survey.

3. Studies that assessed any health outcomes as outcome variables after retirement at baseline and follow-up surveys.

4. Studies that used a prospective cohort design.

5. Studies published in English or Japanese.

6. Studies published in peer-reviewed journals (including advanced online publication).

Exclusion criteria are as follows:

1. Studies targeting participants who have any specific disorder.

2. Studies targeting participants who experienced early retirement due to any problem with their own health, family caregiving responsibilities or other issues compelling participants to retire early.

3. Studies targeting participants who have been fired or laid off by their employer. 


\section{Information sources and search strategy}

A systematic search was conducted on 15 April 2019. Published studies were searched using the following electronic databases: MEDLINE, EMBASE, PsycINFO, PsycARTICLES and Japan Medical Abstracts Society. The search terms included words related to the PECO of the studies (see online supplementary appendix for the details of the search strategy). The search terms were determined based on our previous meta-analyses on the association of psychosocial factors at work with metabolic syndrome $e^{28} 29$ and inflammation ${ }^{30}$, which was an extensive set of terms covering a broad range of psychosocial factors at work (see details of search terms elsewhere ${ }^{28-30}$ ). In order to conduct the literature search comprehensively, a wide range of search terms related to exposure were selected.

The following search terms will be used:

1. Psychosocial factors at work (stress, sedentary, workload, demand, control, effort, reward, support, social capital, working hours and shift work, among others).

2. Retirement (retire, step down, resign, leave, quit and withdraw, among others).

3. Study design (longitudinal, prospective, cohort and follow-up, among others).

\section{Study records}

\section{Data management}

Study records will be managed by using a standardised form in a Microsoft Excel (Washington, USA) file. Prior to screening the studies, deduplication within this Excel file will be conducted by KI.

\section{Selection process}

Fifteen investigators (KI, YA, HA, EA, AI, RI, MI, HE, $\mathrm{YO}, \mathrm{YK}, \mathrm{ASa}, \mathrm{NS}, \mathrm{KT}, \mathrm{AH}$ and $\mathrm{KW}$ ) will independently assess the studies according to the eligibility criteria through the following steps (ie, sifting phase and fulltext review phase). After excluding duplicated records, the remained articles will be shared by 15 investigators, and pairs of investigators will independently assess the title and abstract of each article to identify eligible studies according to the eligibility criteria (sifting phase). In the full-text review phase, pairs of investigators will independently review the full texts that will be included as eligible studies after the sifting phase. When the results (ie, include or exclude) between the pairs of investigators are inconsistent at this phase, the disagreements will be settled by consensus among all authors. The results of the assessment by a pair of the two independent reviewers (ie, consistent or inconsistent) and reasons for excluding studies will be recorded. A flow chart will be provided to show the entire review process. Before starting the sifting phase, a brief session will be held to monitor quality of assessment by each investigator.

\section{Data collection process}

Data will be extracted independently from the included studies by 15 investigators (KI, YA, HA, EA, AI, RI, MI, HE, YO, YK, ASa, NS, KT, AH and KW) using a standardised data extraction form. Any disagreements or inconsistencies will be solved by consultation and consensus among all authors. Investigators will extract data on publication year, study design, country where the study was conducted, the number of participants included in the baseline survey and in the statistical analysis, demographic characteristics of participants (ie, age, sex and occupational status), the number of years from baseline survey to retirement, the number of years from retirement to follow-up surveys, length of follow-up and attrition rate, exposure variables before retirement (ie, adverse psychosocial factors at work), outcome variables after retirement (ie, any physical or mental health indicator) and sufficient data for calculating the coefficients $(\beta, \gamma)$, ORs, relative risks (RRs) or HRs with SEs or 95\% CIs for the association between adverse work-related psychosocial factors before retirement and health outcomes after retirement. If necessary, the authors of the included studies will be contacted to obtain additional relevant information.

\section{Data synthesis}

The included studies will be statistically synthesised in a meta-analysis to estimate pooled coefficients and $95 \%$ CIs, stratified by types of measures of association $(\beta, \gamma, \mathrm{OR}, \mathrm{RR}$ and HR). If the included studies report ORs, RRs or HRs, we will calculate log-transformed ORs, RRs or HRs, and determine SEs based on 95\% CIs. These parameters will be used in the meta-analysis and for examining publication bias by means of a funnel plot and Egger's test.

\section{Risk of bias in individual studies and assessment of meta- bias}

Fifteen investigators (KI, YA, HA, EA, AI, RI, MI, HE, YO, $\mathrm{YK}, \mathrm{ASa}$, NS, KT, AH and KW) will independently assess in pairs the quality of each included study using the internationally recognised tool for evaluating risk of bias (Risk Of Bias In Non-randomised Studies-of Interventions; ROBINS-I). ${ }^{31}$ The ROBINS-I is a newly developed tool for evaluating risk of bias in estimates of the comparative effectiveness (harm or benefit) of interventions (or specific exposures) from studies that did not use randomisation to allocate units (individuals or clusters of individuals) to comparison groups, including observational studies, such as cohort studies and case-control studies. ${ }^{31}$ The risk of bias is classified as low, high or unclear risk. Any discrepancies in the quality assessment among the investigators will be recorded and discussed among all authors, until consensus is reached. For the assessment of meta-bias, publication bias will be assessed by using Egger's test, as well as visually on a funnel plot.

\section{Statistical methods}

\section{Primary analyses}

For the main analysis, we will synthesise all types of psychosocial factors at work and all types of health outcomes. In this review, it is expected that most of the outcomes of studies that will be included are assessed as dichotomous variables. ${ }^{19-22}$ If the outcomes are assessed by continuous 
variables, we will apply the appropriate cut-off points and convert to dichotomous variables. If we cannot use the appropriate cut-off point, dichotomous variables and continuous variables will be analysed separately.

Meta-analysis will be conducted when at least three eligible studies can be collected. If a meta-analysis is not appropriate (ie, only two or fewer studies are eligible and included), the results will be presented in a narrative format. A fixed-effect model will be used if heterogeneity is not observed (eg, types of exposures and populations, among others); otherwise, a random-effects model will be used. ${ }^{32}$ Heterogeneity will be assessed using the $\chi^{2}$ test with Cochran's $Q$ statistic and $\mathrm{I}^{2}{ }^{33}$ Usually, $\mathrm{I}^{2}$ Values of $25 \%, 50 \%$ and $75 \%$ indicate low, medium and high heterogeneity, respectively. ${ }^{34}$

\section{Subgroup and sensitivity analyses}

Subgroup analyses will be conducted to compare the results under specific outcomes or conditions. Major possible grouping characteristics will include types of exposure according to some specific work-related stress models (ie, job strain and support from supervisors/ colleagues based on the job demand control support model, ${ }^{18} 35$ and effort-reward imbalance based on the effort reward imbalance model ${ }^{36}$ ) and outcome (diseases/ symptoms, mobility/physical function and cognitive function). Any subgroup differences will be reported, and our findings will be explained by considering these differences. If trends are observed between pooled associations and any grouping characteristics, meta-regression will be conducted. ${ }^{37}$ A sensitivity analysis will be conducted for included studies where the ROBINS-I is classified as low risk. $^{31}$

\section{Patient and public involvement}

There is no direct patient or public involvement in the design of this study.

\section{ETHICS AND DISSEMINATION}

Results and findings will be submitted and published in a scientific peer-reviewed journal and will be disseminated broadly to researchers and policymakers interested in the translatability of scientific evidence into good practices.

\section{Strengths and limitations}

To our knowledge, this will be the first systematic review and meta-analysis to show integrated evidence for the associations between psychosocial factors at work and postretirement health conditions. The findings of this study will establish a link between psychosocial factors at work in working age and health problems after retirement. Then it would contribute to prevention of chronic conditions and promotion of health and well-being of older adults after retirement, that is, to achieve active ageing in our rapidly ageing society through proposing an innovative life-course strategy to improve psychosocial factors at work in working age. Practically, the results of this study could facilitate implementation of appropriate intervention for workers who have been exposed to specific adverse psychosocial factors at work.

This systematic review and meta-analysis may have some limitations. A major limitation is that this study will include mostly observational studies and will not be limited to randomised controlled trials, although we will focus on well-designed prospective cohort studies. In addition, the findings may be biassed by potential confounders. Moreover, generalisation of the findings may be limited by participants' characteristics, depending on the included studies.

\section{Author affiliations}

${ }^{1}$ Department of Mental Health, Graduate School of Medicine, The University of Tokyo, Tokyo, Japan

${ }^{2}$ Department of Public Health, Kitasato University School of Medicine, Sagamihara, Japan

${ }^{3}$ Center for Public Health Sciences, National Cancer Center Japan, Tokyo, Japan ${ }^{4}$ Department of Psychiatric Nursing, Graduate School of Medicine, The University of Tokyo, Tokyo, Japan

${ }^{5}$ Faculty of Human Sciences, University of Tsukuba, Tokyo, Japan

${ }^{6}$ Department of Public Health, Tokyo Women's Medical University, Tokyo, Japan ${ }^{7}$ School of Health Innovation, Kanagawa University of Human Services, Kawasaki, Japan

${ }^{8}$ Department of Mental Health, Institute of Industrial Ecological Sciences, University of Occupational and Environmental Health, Japan, Kitakyushu, Japan

${ }^{9}$ Faculty of Policy Management, Keio University, Fujisawa, Japan

Contributors KI, AT, YA, HA, EA, Al, RI, MI, HE, YO, YK, ASa, NS, KT, AH, KW, ASh and NK have made substantial contributions to the conception and design, writing the protocol and revising it critically for important intellectual content, and approving the final version to be published.

Funding This study is supported by the Work-related Diseases Clinical Research Grant 2018 (180701) from the Ministry of Health, Labour and Welfare, Japan.

Competing interests None declared.

Patient consent for publication Not required.

Ethics approval Ethical approval will not be needed to apply this review protocol because data will be extracted from the published studies and there will be no concerns about privacy.

Provenance and peer review Not commissioned; externally peer reviewed.

Open access This is an open access article distributed in accordance with the Creative Commons Attribution Non Commercial (CC BY-NC 4.0) license, which permits others to distribute, remix, adapt, build upon this work non-commercially, and license their derivative works on different terms, provided the original work is properly cited, appropriate credit is given, any changes made indicated, and the use is non-commercial. See: http://creativecommons.org/licenses/by-nc/4.0/.

\section{REFERENCES}

1. United Nations. World population prospects: the 2015 revision United Nations Department of Economic Social Affairs; 2015: 1-66.

2. GBD 2015 Disease and Injury Incidence and Prevalence Collaborators. Global, regional, and national incidence, prevalence, and years lived with disability for 310 diseases and injuries, 19902015: a systematic analysis for the global burden of disease study 2015. Lancet 2016;388:1545-602.

3. Marengoni A, Angleman S, Melis R, et al. Aging with multimorbidity: a systematic review of the literature. Ageing Res Rev 2011;10:430-9.

4. Sheffield KM, Peek MK. Changes in the prevalence of cognitive impairment among older Americans, 1993-2004: overall trends and differences by race/ethnicity. Am J Epidemiol 2011;174:274-83.

5. Clegg A, Young J, lliffe S, et al. Frailty in elderly people. Lancet 2013;381:752-62.

6. Steptoe A, Deaton A, Stone AA. Subjective wellbeing, health, and ageing. The Lancet 2015;385:640-8. 
7. World Health Organization. World report on ageing and health; 2015.

8. Kalache A, Kickbusch I. A global strategy for healthy ageing. World health 1997;50:4-5.

9. Liu S, Jones RN, Glymour MM. Implications of lifecourse epidemiology for research on determinants of adult disease. Public Health Rev 2010;32:489-511.

10. Stuck AE, Walthert JM, Nikolaus T, et al. Risk factors for functional status decline in community-living elderly people: a systematic literature review. Soc Sci Med 1999;48:445-69.

11. Michel J-P, Newton JL, Kirkwood TBL. Medical challenges of improving the quality of a longer life. JAMA 2008;299:688-90.

12. Rizzuto D, Fratiglioni L. Lifestyle factors related to mortality and survival: a mini-review. Gerontology 2014;60:327-35.

13. Burton J. WHO healthy workplace framework and model: background and supporting literature and practices World Health Organization; 2010.

14. WHO Commission on Social Determinants of Health. Closing the gap in a generation: health equity through action on the social determinants of health: Commission on social determinants of health final report World Health Organization; 2008.

15. Topa G, Depolo M, Alcover C-M. Early retirement: a meta-analysis of its antecedent and subsequent correlates. Front Psychol 2017:8:2157.

16. Schaap R, de Wind A, Coenen P, et al. The effects of exit from work on health across different socioeconomic groups: a systematic literature review. Soc Sci Med 2018;198:36-45.

17. International Labour Office. Psychosocial factors at work: recognition and control: report of the joint ILO/WHO Committee on occupational health, ninth session, Geneva, 18-24 September 1984; 1986.

18. Karasek RA. Job demands, job decision latitude, and mental strain: implications for job redesign. Adm Sci Q 1979;24:285-308

19. Nilsen C, Andel R, Fors S, et al. Associations between work-related stress in late midlife, educational attainment, and serious health problems in old age: a longitudinal study with over 20 years of follow-up. BMC Public Health 2014;14.

20. Sabbath EL, Glymour MM, Descatha A, et al. Biomechanical and psychosocial occupational exposures: joint predictors of postretirement functional health in the French GAZEL cohort. Adv Life Course Res 2013;18:235-43.

21. Virtanen M, Ferrie JE, Batty GD, et al. Socioeconomic and psychosocial adversity in midlife and depressive symptoms post retirement: a 21-year follow-up of the Whitehall II study. Am J Geriatr Psychiatry 2015;23:99-109.

22. Wahrendorf M, Blane D, Bartley M, et al. Working conditions in mid-life and mental health in older ages. Adv Life Course Res 2013;18:16-25.
23. Andel R, Infurna FJ, Hahn Rickenbach EA, et al. Job strain and trajectories of change in episodic memory before and after retirement: results from the health and retirement study. J Epidemiol Community Health 2015:69:442-6.

24. Theorell T, Hammarström A, Aronsson G, et al. A systematic review including meta-analysis of work environment and depressive symptoms. BMC Public Health 2015;15:738.

25. Theorell T, Jood K, Järvholm LS, et al. A systematic review of studies in the contributions of the work environment to ischaemic heart disease development. Eur J Public Health 2016;26:470-7.

26. Moher D, Shamseer L, Clarke M, et al. Preferred reporting items for systematic review and meta-analysis protocols (PRISMA-P) 2015 statement. Syst Rev 2015;4.

27. Stroup DF, Berlin JA, Morton SC, et al. Meta-Analysis of observational studies in epidemiology: a proposal for reporting. meta-analysis of observational studies in epidemiology (moose) group. JAMA 2000;283:2008-12.

28. Sakuraya A, Watanabe K, Kawakami N, et al. Work-Related psychosocial factors and onset of metabolic syndrome among workers: a systematic review and meta-analysis protocol. BMJ Open 2017:7:e016716.

29. Watanabe K, Sakuraya A, Kawakami N, et al. Work-Related psychosocial factors and metabolic syndrome onset among workers: a systematic review and meta-analysis. Obes Rev 2018;19:1557-68.

30. Eguchi H, Watanabe K, Kawakami N, et al. Psychosocial factors at work and inflammatory markers: protocol for a systematic review and meta-analysis. BMJ Open 2018;8:e022612.

31. Sterne JAC, Hernán MA, Reeves BC, et al. ROBINS-I: a tool for assessing risk of bias in non-randomised studies of interventions. BMJ 2016;355

32. Hunter JE, Schmidt FL. Fixed effects vs. random effects metaanalysis models: implications for cumulative research knowledge. Int Journal Select Assess 2000;8:275-92.

33. Higgins JPT, Thompson SG. Quantifying heterogeneity in a metaanalysis. Stat Med 2002;21:1539-58

34. Higgins JP, Green S. Cochrane handbook for systematic reviews of interventions. John Wiley \& Sons, 2011

35. Johnson JV, Hall EM. Job strain, work place social support, and cardiovascular disease: a cross-sectional study of a random sample of the Swedish working population. Am J Public Health 1988;78:1336-42.

36. Siegrist J. Adverse health effects of high-effort/low-reward conditions. J Occup Health Psychol 1996;1:27-41.

37. Thompson SG, Higgins JPT. How should meta-regression analyses be undertaken and interpreted? Stat Med 2002;21:1559-73. 